Claudio Chiuderi

Istituto di Astronomia, Università di Firenze, Italy.

\title{
INTRODUCTION
}

High energy phenomena in the solar physics context, simply means solar flares. To be sure, the energies attained during flares are certainly not very impressive on a cosmic-ray scale. The most energetic particles belong the $\mathrm{GeV}$ range, the highest temperatures are of the order of $10^{7} \mathrm{~K}, \gamma$-ray emission is occasional and the total energy emitted remains below $10^{33}$ ergs for all the flares so far observed. Apart from an absolute energy scale, flares are also energetically irrelevant on a solar scale. In fact in a large flare a few units in $10^{32}$ ergs are emitted, with a total duration of about one hour and a total surface area involved of a few units in $10^{-4}$ of the solar surface. Recalling the values of the luminosity, $\mathrm{L}_{\odot} \simeq 4 \times 10^{33} \mathrm{erg} \mathrm{s}^{-1}$ and the solar flux $\mathrm{F}_{\odot} \simeq 6.3 \times 10^{10} \mathrm{erg} \mathrm{cm}^{-2} \mathrm{~s}^{-1}$, we see that

$$
\mathrm{L}_{\mathrm{flare}} \simeq 10^{-5} \mathrm{~L}_{\odot} \text { and } \mathrm{F}_{\mathrm{flare}} \simeq\left(10^{-1}-10^{-2}\right) \mathrm{F}_{\odot} \text {. }
$$

In spite of their irrelevance to the Sun's global energetics, flares are an important and fascinating phenomenon, not only for solar physics but for the whole of astrophysics as well. The reason is that they most likely are a small-scale model of a class of phenomena widespread in the astrophysical Universe: the violent conversion of some form of energy into heat and kinetic energy of accelerated particles. As we shall see, it can be argued that in flares the primary energy supply comes from previously stored magnetic energy. Flares occur relatively nearby and can be observed to a reasonable degree of detail. We can hope, therefore, to be able to identify the key processes responsible for the transformation of magnetic energy in other forms of energy. Nobody can be sure that the information gained through the study of flares can be safely scaled to other astrophysical situations, but this appears to be a sensible first step toward a proper quantitative understanding of one of the basic astrophysical processes.

In the following I shall briefly outline the most important obser367

G. Setti, G. Spada, and A. W. Wolfendale (eds.), Origin of Cosmic Ray's, $367-372$.

Copyright $=1981$ by the IAU. 
vational aspect of solar flares as we know them today, and concentrate then on the mechanisms of primary energy release.

\section{FLARE OBSERVATIONS}

In the following the word flare is meant to indicate the set of high-energy events that involve the presence of suprathermal particles, the emission of radiation whose brightness temperature exceeds the local kinetic temperature and the ejection of large masses of plasma from the solar atmosphere.

Flares are observed over the whole electromagnetic spectrum, which implies that a large vertical portion of the solar atmosphere is involved. However, they are seldom seen in white light, a direct result of the low flare luminosity as compared with the total solar output. Observations show that the continuum of the solar spectrum is hardly affected by flares, while the most prominent absorption lines show strong temporal variations, thus proving the chromospheric rather than photospheric nature of the optical flare. In the temporal development of a flare, three phases are usually recognized. A precursor phase, where a slowly rising intensity is observed mainly in EUV and soft $\mathrm{X}$-rays and at microwave radio frequencies. Then an impulsive or flash phase follows, characterized by intense emission at practically all wavelengths. Finally we have the gradual or main phase, where the activity decays over varied timescales. Typically the impulsive phase may last $10^{2} \mathrm{~s}$ and the gradual phase $10^{3} \mathrm{~s}$. Emission in the EUV, hard $\mathrm{X}$-rays and microwave is mainly concentrated in the impulsive phase.

As a result of observations from space our global knowledge of the flare phenomenon has considerably improved. A major achievement of the Skylab Mission has been the recognition of the importance of the coronal aspect of the flares and their close association with the solar magnetic field structure. In the light of these new observations the chromospheric flare, the only one once known, must be considered rather a consequence of processes that are initiated in the corona. Coronal flares occur almost exclusively in arch-like structures

(coronal loops). The loop is filled with coronal plasma ( $\simeq \simeq 1-2 \times 10^{6} \mathrm{~K}$ ) and links two low-temperature $\left(\mathrm{T} \simeq 1-5 \times 10^{4} \mathrm{~K}\right)$ chromospheric "ribbons", belonging to oppositely polarized magnetic regions. During the flare's impulsive phase a hot plasma cloud $\left(\mathrm{T} \approx 2 \times 10^{7} \mathrm{~K}\right)$ is formed at the apex of the loop. The plasma then cools and becomes clearly visible in many intermediate temperature $\left(\mathrm{T} \simeq 2 \times 10^{6} \mathrm{~K}\right.$ ) coronal lines (post-flare loops). The loops supposedly delineate the gross magnetic field structure. Sometimes they can be observed before the occurence of the flare. They appear to be relatively long-lived structures, with 1 ifetimes of hours to more than one day. Apart from electromagnetic radiation, a major manifestation of flares is the emission of high-energy particles. Acceleration of electrons and ions to $100 \mathrm{KeV}$ is a standard feature, but the so-called "proton flares" produce protons up to $100 \mathrm{MeV}$ and the "cosmic-ray flares" may boost heavy particles in the Gev range. 
By interacting with the ambient nuclei in the solar atmosphere, these high energy particles can initiate nuclear reactions. This may result in $\gamma$-ray emission via the radiative decay of excited nuclei, or positron annihilation, or deuterium formation. Observations have been successfully performed of the $2.23 \mathrm{MeV}$ line from $p+n \rightarrow d+\gamma$, of the $0.51 \mathrm{MeV}$ line from $\mathrm{e}^{+}+\mathrm{e}^{-} \rightarrow \gamma+\gamma$ and of the $4.43 \mathrm{MeV}$ and $6.14 \mathrm{MeV}$ de-excitation 1 ines of $\mathrm{C}^{12}$ and $\mathrm{O}^{16}$. Another energetically important aspect of flares is the ejection of large masses of plasma $\left(M \simeq 10^{16} \mathrm{~g}\right)$. The kinetic energy associated with the ejecta represents about one half of the flare energy.

This sketchy description of the most relevant observable features of flares, is already sufficient to show the complexity of the phenomenon. The theoretical interpretationsdo not attempt a detailed description but rather try to identify the basic physical processes responsible for the great variety of the observed effects.

\section{FLARE PHYSICS}

The minimum set of questions that any flare theory should answer is the following:

i) Which are the energy build-up mechanisms?

ii) Which are the energy release mechanisms?

As already anticipated, there is a general consensus on the fact that energy is stored in magnetic form prior to the flare. Alternative sources, such as gravitational or thermal energy, fall short of orders of magnitude, especially in the case of large flares. On the contrary, there are in principle no difficulties in fulfilling the energy requirements with magnetic energy: a field of $160 \mathrm{G}$, occupying a volume of $10^{29} \mathrm{~cm}^{3}$, has an energy of $10^{32}$ ergs. Of course the type of field must be specified. In fact potential (i.e.current-free) fields are of no interest here, since they constitute the minimum energy configuration. Thus, if the energy storage mode is magnetic, electrical currents must flow in the solar corona. The presence of currents is also to be expected on different grounds. In fact, the magnetic field lines threading the solar corona must be rooted in the much denser photospheric layers. Given the high electrical conductivity of the solar atmosphere, the field is effectively frozen-in. Thus any motion in the high- $\beta$ photospheric plasma inevitably induces magnetic stresses, or equivalently currents, that propagate in the low- $\beta$ coronal plasma. According to this picture, the ultimate energy source for flares resides in those (turbulent) photospheric or sub-photospheric motions that are responsible for the generation of currents. The subject of the dynamical evolution of magnetic fields, especially force-free fields, has recently received a great deal of attention, in spite of the considerable degree of mathematical difficulty involved. Solutions can be found that prove the existence of a critical stress: if the initial magnetic configuration is stressed beyond a certain level a catastrophic rear- 
rangement of the field takes place. This is highly suggestive of the abrupt onset of a physical flare, but a convincing correlation between this rather abstract treatment and the observed properties remains to be made. The above mentioned studies have however shown one thing: the accumulation of magnetic energy appears to be a plausible assumption on general ground, but a direct verification of the validity of the proposed mechanisms requires a knowledge of the magnetic field structure and evolution well beyond present-day possibilities.

If the stored energy is magnetic, we must still explain how this energy is released, and how it is released on a rather fast timescale. The main problem here comes from the fact that the decrease of magnetic energy implies the destruction of part of the magnetic field and this is by no means easy in a highly conductive plasma, like the solar one. The basic equation that governs the changes of the magnetic field is :

$$
\frac{\partial \underline{\mathrm{B}}}{\partial \mathrm{t}}=\underset{\sim}{\nabla} \mathrm{x}(\underset{\sim}{\mathrm{v}} \times \underset{\sim}{\mathrm{B}})+\eta \nabla^{2} \underset{\sim}{\mathrm{B}}
$$

where $n=c^{2} /(4 \pi \sigma)$, $\sigma$ being the electrical conductivity. The above equation displays two characteristic timescales, depending on the ratio of the two terms on the right hand side, i.e. on the magnetic Reynolds number $S$ :

$$
\mathrm{S}=\mathrm{L} \mathrm{v} / \mathrm{n}
$$

At large $S$ the first term dominates, the fields are frozen-in and the typical timescale is the Alfvén transit time

$$
\tau_{\mathrm{a}}=\mathrm{L} / \mathrm{v}=\mathrm{L} / \mathrm{C}_{\mathrm{A}}
$$

since in this situation the velocity scale is given by the Alfvén speed, $c_{A}$. At low $S$, the field changes are due to resistive diffusion, or in other words to the ohmic dissipation of the sustaining currents. The timescale is then

$$
\tau_{r}=L^{2} / \eta
$$

In solar situations $\mathrm{S}>10^{9}, \tau_{\mathrm{a}}$ is of the order of seconds and $\tau_{\mathrm{r}}$ of the order of $10^{2}$ years. Neither of these two timescales is suitable for the description of a flare, whose characteristic time lies in between. It is clear however that the resistive dissipation of the magnetic field is the most direct, and possibly the only, mechanisms for magnetic energy conversion. We are thus faced with a two-fold problem: to locate in the high-S solar corona the places where the resistive term can play a role and to reduce $\tau_{r}$ by many orders of magnitude.

To answer the first question let us consider an incompressible case and rewrite the magnetic induction equation in the form 


$$
\frac{\partial \underset{\sim}{B}}{\partial t}=(\underset{\sim}{v} \cdot \underset{\sim}{\nabla}) \underset{\sim}{B}-(\underset{\sim}{B} \cdot \underset{\sim}{\nabla}) \underset{\sim}{v}+r_{i} \nabla^{\prime} \underset{\sim}{B}
$$

In plane geometry, $\underset{\sim}{B}=B_{y}(x) \underset{\sim}{e}+B_{z}(x) \underset{z}{e_{z}}$ and the time variation of the initially vanishing component ${ }^{2} B_{X}$ (the "reconnecting" field) is given by

$$
\omega B_{x}^{\prime}=-i(\underset{\sim}{(k}) v_{x}+n\left(\frac{\partial^{2}}{\partial x^{2}}-k^{2}\right) B_{x} .
$$

Here we assumed $B_{x} \sim \exp (i \underset{\sim}{k} \cdot \underline{x}+\omega t)$ and $\underset{\sim}{k}=k_{y} \underset{\sim}{e}+k_{z} \underset{z}{e}$. From Eq. (3) we see that the resistive term will dominate wherever $k \cdot B \approx 0$. Thus the location of the "resistive" layer depends on $\mathrm{k}$, which in turn is controlled by the boundary conditions. The width of the resistive layer is determined by the rate of change of $\mathrm{k} \cdot \mathrm{B}$, or in other words by the degree of shear of the field. Growing perturbations $(\omega>0)$ can be found'when the last term of Eq. (3) is positive. For a highly sheared field, the resistive sheet width, $l$, will be much smaller than the typical linear dimension of the system, L. Thus the resistive time $\tau_{r} \simeq \ell^{2} / n \ll L^{2} / n$. Outside the resistive layer the field is effectively frozen-in and is convected towards the dissipative region by the fluid motion, where the plasma and the magnetic field can decouple. The magnetic lines then tear and reconnect, forming elongated cells or "islands".

There are a number of features of this scheme of dynamical reconnection that makes it particularly attractive for application to solar flares, especially when compared with the more familiar process of stationary reconnection at neutral points. First of all the field need not vanish anywhere, but must simply possess a certain degree of shear. Second, the tearing-mode instability is a spontaneously growing perturbation: the flow pattern and the field distorsion result from the mutual plasmafield interaction without any need to impose artificial external conditions. Finally, the location of the reconnecting process is not fixed a priori but is chosen by the perturbation itself. From this considerations we see that it is rather difficult to make general statements about the efficiency or the speed of the tearing-mode instability, since they depend crucially on the detailed geometry of the magnetic field.

Again, the solution of the primary energy release in flares seems to be tied to the measurement of coronal magnetic fields. Such measurements have not been possible so far. The only chance in a near future seems to reside in polarimetry in the EUV range. First-generation instruments of this kind are part of the instrumentation of the Solar Maximum Mission, second-generation are being planned to fly on Spacelab. As a conclusion, it is fair to say that flare studies have not yet succeeded in pinning 
down the mechanism that governs this complex phenomenon. However, even if the final answers to the basic questions listed at the beginning of this Section have not been given, it has been possible to identify a few firm physical points that will certainly be the basis for the theory of solar flares.

\section{References}

The literature on solar flares is very vast. We list here a few recent review papers or books to which we refer for an extensive bibliography.

Kane, S.R.: 1975, Solar Gamma, X and EUV Radiation, Reidel Publ. Co. Dordrecht, Holland.

Priest, E.R. (Ed.): 1980, Solar Flare MHD, Gordon and Breach, New York, USA (to appear).

Rust, D.M.. 1980, Solar System Plasma Physics, (C.F. Kennel, L.J. Lanzerotti and E.N. Parker, Eds.),North-Holland, New York, USA.

Sakurai, K.: 1974, Astrophys. Space Sci. 28, 375.

Sturrock, P.A. (Ed.): 1980, Solar Flares, Proceedings of the Second Skylab Workshop, Univ. of Colorado Press, Boulder, USA.

Svestka, Z.: 1976, Solar Flares, Reidel Pub1. Co. Dordrecht, Holland.

Tandberg-Hanssen, E.: 1967, Solar Activity, Blaisde11, Waltham, USA. 